\title{
Early-Time Behavior of the Ground Transient Resistance of Overhead Lines
}

\author{
S. Loyka, A. Kouki \\ Department of Electrical Engineering \\ Ecole de Technologie Superieure \\ 1100, Notre-Dame St. West \\ Montreal (Quebec), H3C 1K3, CANADA \\ Email: sergey.loyka@ieee.org
}

\begin{abstract}
Ground resistance gives a substantial contribution to the transient response of overhead transmission lines. While the late-time behaviour of the ground transient resistance has been understood quite well, the same cannot be said of early-time behaviour. In this paper, we investigate the early-time behaviour using a high-frequency expansion of the ground impedance in a Taylor series and derive a compact closed-form analytical expression for it. This expression can be used for the transient analysis of transmission lines in a computationally efficient way.
\end{abstract}

\section{INTRODUCTION}

As it has been widely recognized, ground impedance has a profound effect on the transient response of overhead transmission lines to external disturbances like lightning discharges $[1,2]$. This impedance is dispersive in nature, i.e., time and frequency dependent and an accurate prediction of transient phenomena on the lines depends heavily on the accuracy of its model. While several closed-form ground impedance models are available for the overhead transmission line in the frequency domain, only numerical or grossly approximate closed-form models are available in the time domain. Given that transient analyses are carried out in the time-domain, the ground impedance (frequency-domain function) must be transformed into the time domain giving the "ground transient resistance" function, which is the quantity used for the analysis of the transmission line response. Unfortunately, it is not possible to obtain an analytical form of the transient resistance in the general case and one must resort to numerical techniques for accurate prediction of the transient behaviour over extended time intervals. This results in a computationally intensive process with little if any insight into the physics of the problem.

In order circumvent the computational limitation and to gain some physical insight into the effect of ground impedance on the transient response of overhead lines at various time intervals, it is preferable to have an analytical closed-form representation of the ground transient resistance. In this context, the low-frequency approximation of the ground impedance is employed, which, as was first demonstrated by Timotin [3], allows one to obtain a closed-form analytical representation of the transient resistance. While this results in significant savings in computational resources and despite its wide acceptance, this approach has a major drawback: the early-time behaviour of the transient impedance is incorrectly predicted [4]. In fact, the low-frequency approximation gives a divergence of the kind $1 / \sqrt{t}$ for $t \rightarrow 0$ [1]. Further more, the general model (without low-frequency approximation) predicts that the transient resistance $\rightarrow$ constant for $t \rightarrow 0$ [4]. Although this divergence does not present a problem for numerical analysis $[1,5]$, it gives the wrong physical picture and may result in inaccurate predictions.

Thus, the study of the early-time behaviour is of significant importance for accurate analysis of transient phenomena in overhead transmission lines. In this paper, we further develop the approach reported in [4] and show that, using the highfrequency expansion of the ground impedance in Taylor series, it is possible to obtain a compact closed-form analytical expression for the early-time behaviour of the ground transient resistance, which can be further used for the transient analysis of transmission lines in a computationallyefficient way. Detailed analysis of the accuracy of high and low-frequency approximations show that none of these approximations provides a satisfactory accuracy in the "intermediate" region (i.e., when the time is already not "early" but still not "late"). This region is the most difficult one to predict accurately because neither approximation alone (high frequency or low frequency) gives satisfactory results. The size and position of this region depends on the transmission line geometry and the earth electrical parameters (dielectric constant and conductivity). Thus, some combination of these two approximations is required for accurate predictions in this region.

\section{GROUND IMPEDANCE AND ITS LOW-F REQUENCY APPROXIMATION}

Per-unit-length ground impedance in the frequency domain for a single wire located at height $h$ above ground within the transmission line approximation (i.e., neglecting the radiation effect) is [1, 2]: 


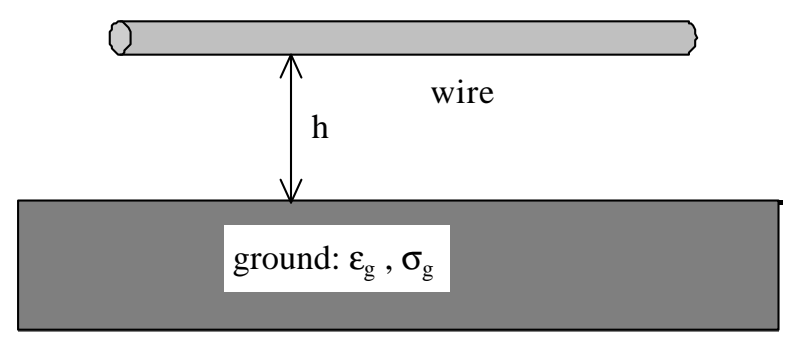

Figure 1. Overhead transmission line scenario.

$$
Z_{g}^{\prime}(\omega)=\frac{j \omega \mu_{0}}{\pi} \int_{0}^{\infty} \frac{e^{-2 h x}}{\sqrt{x^{2}+\gamma_{g}^{2}}+x} d x
$$

where $\gamma_{g}$ is the propagation constant in the ground,

$$
\gamma_{g}=\sqrt{j \omega \mu_{0} \sigma_{g}-\omega^{2} \mu_{0} \varepsilon_{g}}
$$

$\sigma_{g}$ - is the ground conductivity, $\varepsilon_{g}$ - is the ground permittivity, $\mu_{0}$ - is the permeability of the free space. The electric field component due to the ground impedance is

$$
E_{g}(\omega)=Z_{g}^{\prime}(\omega) \cdot I(\omega)
$$

where $I(\omega)$ is the line current. In the time domain, this equation takes the following form:

$$
e_{g}(t)=z_{g}(t) * i(t)
$$

where $z_{g}(t)$ denotes the inverse Fourier transform of $Z_{g}^{\prime}(\omega)$, and "*" denotes the convolution. The so-called ground transient resistance, which is given by the inverse Fourier transform,

$$
\xi(t)=\boldsymbol{F}^{-1}\left(\frac{Z_{g}^{\prime}}{j \omega}\right),
$$

can also be used for $e_{g}(t)$ calculation,

$$
e_{g}(t)=\xi(t) * \frac{d i(t)}{d t}
$$

The term "ground transient resistance" is used for $\xi(t)$ because it is a time-domain function and its dimension is Ohms/meter [2, 6]. For a low-frequency approximation, when

$$
\omega<<\omega_{\max }=\frac{\sigma_{g}}{\varepsilon_{g}},
$$

and only the first term in (2) is essential, $\xi(t)$ can be calculated by Timotin's formula [1-3]

$$
\xi(t)=\frac{\mu_{0}}{\pi \tau_{g}}\left[\frac{1}{2 \sqrt{\pi}} \sqrt{\frac{\tau_{g}}{t}}+\frac{1}{4} \exp \left(\frac{\tau_{g}}{t}\right) \operatorname{erfc}\left(\sqrt{\frac{\tau_{g}}{t}}\right)-\frac{1}{4}\right],
$$

where $\tau_{g}=h^{2} \mu_{0} \sigma_{g}$, and $\operatorname{erfc}(x)$ is the complementary error function

$$
\operatorname{erfc}(x)=\frac{2}{\sqrt{\pi}} \int_{x}^{\infty} e^{-t^{2}} d t .
$$

As it is pointed out in $[1,5,6],(8)$ has singular behavior at $t=0$, and

$$
\xi(t) \approx \frac{\mu_{0}}{2 \pi} \frac{1}{\sqrt{\pi \tau_{g} t}} \text { for } \mathrm{t} \rightarrow 0 .
$$

But the following question arises: is this singularity a physical one or is it a consequence of the low frequency approximation ? As it has been shown in [4], the source of this singularity is the low-frequency approximation and it does not exist if $\xi(t)$ is calculated without the low-frequency assumption. Below we consider this issue in more detail.

\section{TIME-DOMAIN LIMITATION OF THE LOW-F REQUENCY APPROXIMATION}

Due to the fundamental property of the Fourier transform, (8) is not valid for small values of $t$ because it has been obtained in the low-frequency approximation [4]. We will explain it using the following example [6, p.189]. Let us consider the inverse Fourier transform of the frequency-domain function $Y(\omega)$,

$$
y(t)=\frac{1}{2 \pi} \int_{-\infty}^{\infty} Y(\omega) e^{j \omega t} d \omega
$$

If $Y(\omega)$ is a constant, then $y(t)=0$ for all $t \neq 0$ (due to the oscillating factor $e^{j \omega t}$ in the integrand in (11)). If $Y(\omega)$ is not a constant but it varies slowly enough in comparison to $e^{j \omega t}$, then the infinite integration limits can be reduced to the finite ones since the integration subinterval for which $|\omega| t>>1$ does not give a substantial contribution to the total integral due to the fast-oscillating factor $e^{j \omega t}$; the subinterval for which $|\omega| t<1$ does give a substantial contribution since the factor $e^{j \omega t}$ does not oscillate in this subinterval. Further we will carry out simple order-of-magnitude calculations. Taking into account the considerations given above, (11) can be reduced to

$$
y(t) \approx \frac{1}{2 \pi} \int_{-\omega_{\max }}^{\omega_{\max }} Y(w) e^{j \omega t} d \omega .
$$

where

$$
\omega_{\max } \approx \frac{1}{t} .
$$

Thus, the spectrum of $y(t)$ must be known up to $\omega_{\max } \approx 1 / t_{0}$ in order to calculate $y(t)$ at the point $t_{0}$. In a similar manner, if we know the function $Y(\omega)$ up to $\omega_{\max }$, then we can calculate $y(t)$ for $t \geq 1 / \omega_{\max }$. If $y(t)$ must be calculated for a smaller value of $t, Y(\omega)$ has to be known for a larger value of $\omega$. 
Let us now turn our attention to $\xi(t)$. Since $Z_{g}(\omega)$ in the low frequency approximation is known up to $\omega_{\max }=\sigma_{g} / \varepsilon_{g}$ (see Eq. (7)), we can correctly calculate $\xi(t)$ using Eq.(8) for

$$
t \geq t_{\min }=\frac{\varepsilon_{g}}{\sigma_{g}} .
$$

The calculation of $\xi(t)$ using Eq.(8) for $t<t_{\min }$ does not produce a valid result. In this region, we have to use Eq. (1) without the low-frequency approximation.

\section{Ground Transient ResistanCE IN THE EARLY-Time REGION}

First of all, we note that the exact value of $\lim _{t \rightarrow 0} \xi(t)$ (within the transmission line approximation) can be calculated using the initial value theorem for the Laplace transform [4]. According to this theorem,

$$
\lim _{t \rightarrow 0} x(t)=\lim _{s \rightarrow \infty} s X(s)
$$

where $x(t)$ is a time-domain function, $X(s)$ - is its Laplace transform. It is known [2] that for $\omega \rightarrow \infty$

$$
Z_{g}^{\prime}(\omega) \rightarrow Z^{\prime}{ }_{\lim }=\frac{1}{2 \pi h} \sqrt{\frac{\mu_{0}}{\varepsilon_{g}}}
$$

Using (1), (2), (15) and (16), one obtains

$$
\lim _{t \rightarrow 0} \xi(t)=Z^{\prime} \lim =\frac{1}{2 \pi h} \sqrt{\frac{\mu_{0}}{\varepsilon_{g}}}
$$

Thus, we know the ground transient resistance for $t=0$ and need to estimate its value for the small value of time, i.e. for

$$
t<t_{\min }=\frac{\varepsilon_{g}}{\sigma_{g}} .
$$

The obvious solution is to use the linear approximation for $\xi(t)$ between the points $t=0$ (Eq. (17)) and $t=t_{\min }$ (Eq. (8)). However, a more accurate approximation can be obtained using the following approach. First, we transform (1) to the following form:

$$
Z_{g}^{\prime}(\omega)=\frac{j \omega \mu_{0}}{\pi \gamma_{g}^{2}}\left(I_{1}-I_{2}\right)
$$

where

$$
\begin{gathered}
I_{1}=\gamma_{g} \int_{0}^{\infty} e^{-2 h x} \sqrt{1+\left(\frac{x}{\gamma_{g}}\right)^{2}} d x, \\
I_{2}=\int_{0}^{\infty} e^{-2 h x} x d x=\frac{1}{4 h^{2}}
\end{gathered}
$$

In the high-frequency region,

$$
\left|\gamma_{g}\right|>\frac{1}{h}
$$

we expand the square root in (20) in the Taylor series and perform the integration,

$$
I_{1}=\frac{\gamma_{g}}{2 h}\left(1+\sum_{k=1}^{\infty}(-1)^{k+1} \frac{(2 k-3) ! !(2 k-1) ! !}{\left(2 h \gamma_{g}\right)^{2 k}}\right)
$$

Then, the second-order approximation of $Z^{\prime}{ }_{g}(\omega)$ is (keeping only $I_{2}$ and the first term in (23)):

$$
Z_{g}^{\prime}(\omega) \approx \frac{j \omega \mu_{0}}{2 \pi}\left(\frac{1}{h \gamma_{g}}-\frac{1}{2\left(h \gamma_{g}\right)^{2}}\right)
$$

Using (5), one obtains [7]:

$$
\begin{gathered}
\xi(t) \approx Z^{\prime}{ }_{\lim } \exp \left(-\frac{t}{2 t_{\min }}\right) I_{0}\left(\frac{t}{2 t_{\min }}\right)- \\
\frac{1}{4 \pi h^{2} \sigma_{g}}\left(1-\exp \left(-t t_{\min }\right)\right)
\end{gathered}
$$

where $I_{0}$ is the modified Bessel function of the first kind. Let us now consider the range of validity of this approximation. (22) can be transformed to

$$
\omega \mu_{0} \varepsilon_{g} \sqrt{\omega^{2}+\omega_{\max }^{2}}>h^{-2}
$$

Based on this inequality, one obtains the following:

$$
\omega>\omega_{\lim }=\sqrt{2} \omega_{1}\left(1+\sqrt{1+4\left(\frac{\omega_{2}}{\omega_{\max }}\right)^{4}}\right)^{-1 / 2}
$$

where

$$
\omega_{1}=\frac{1}{h^{2} \mu_{0} \sigma_{g}}, \quad \omega_{2}=\frac{1}{h \sqrt{\mu_{0} \varepsilon_{g}}}
$$

(27) can be further reduced to the following approximate expression:

$$
\omega>\omega_{\lim } \approx \max \left[\omega_{1}, \omega_{2}\right]
$$

This is the range of validity of Eq. (24). Going back to the time domain, one may expect that Eq.(25) is valid provided that:

$$
t<t_{\lim }=\omega_{\lim }^{-1}
$$

Thus, the ground transient resistance can be estimated by Eq. (25) for $0 \leq t<t_{\text {lim }}$ and by the Timotin's formula (Eq. (8)) for $t_{\min } \leq t<\infty$.

Fig. 2 and Fig. 3 compare the magnitude and phase correspondingly of the ground impedance computed by (1) with the $1^{\text {st }}$ and $2^{\text {nd }}$ order approximations (Eq. (24) with one and two terms correspondingly). The $1^{\text {st }}$ order approximation provides better accuracy over the entire frequency range, and the $2^{\text {nd }}$ order approximation provides better accuracy in the high-frequency region (however, its accuracy in the low frequency region is worse). 


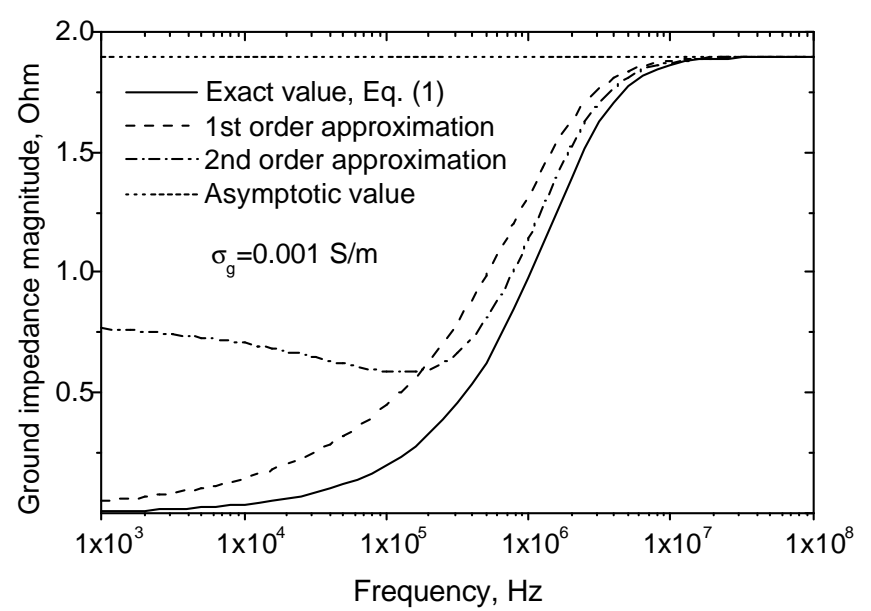

Figure 2a. Ground impedance magnitude versus frequency by Eq. (1), (24) $\left(1^{\text {st }}\right.$ and $2^{\text {nd }}$ order approximations) and its asymptotic value (Eq. (16)); $\varepsilon / \varepsilon_{g}=10, \sigma_{g}=0.001 \mathrm{~S} / \mathbf{m}, h=10 \mathbf{m}$.

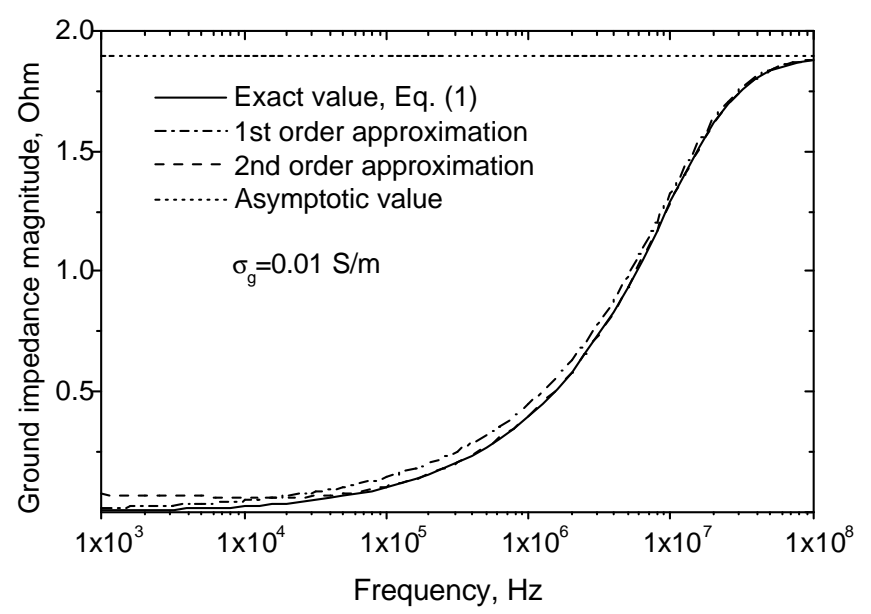

Figure 2b. Ground impedance magnitude versus frequency, $\sigma_{g}=0.01 \mathrm{~S} / \mathrm{m}$.

Thus, one may expect that the $2^{\text {nd }}$ order approximation will provide better accuracy in the early-time region. Note that the accuracy of (24) is much better for higher ground conductivity, especially for the ground impedance magnitude (Fig. 2b). Fig. 4 compares the ground transient resistance computed by (1) and (5) (no low frequency approximation is used) using numerical FFT, by (25) keeping one $\left(1^{\text {st }}\right.$ order approximation) and two ( $2^{\text {nd }}$ order approximation) terms, by Timotin's formula (8) and the asymptotic value (17). For, $\sigma_{g}=0.001 \mathrm{~S} / \mathrm{m}, t_{\min }=8.8 \cdot 10^{-8}$ and $t_{\mathrm{lim}}=1.5 \cdot 10^{-7}$, and for $\sigma_{g}=0.01 \mathrm{~S} / \mathrm{m}, t_{\min }=8.8 \cdot 10^{-9}$ and $t_{\lim }=1.5 \cdot 10^{-6}$. Eq. (25) provides quite a good approximation for the early-time region. However, it is not accurate for later-time region for low ground conductivity $\left(\sigma_{g}=0.001 \mathrm{~S} / \mathrm{m}\right)$, especially the $2^{\text {nd }}$ order approximation. But Timotin's formula provides an

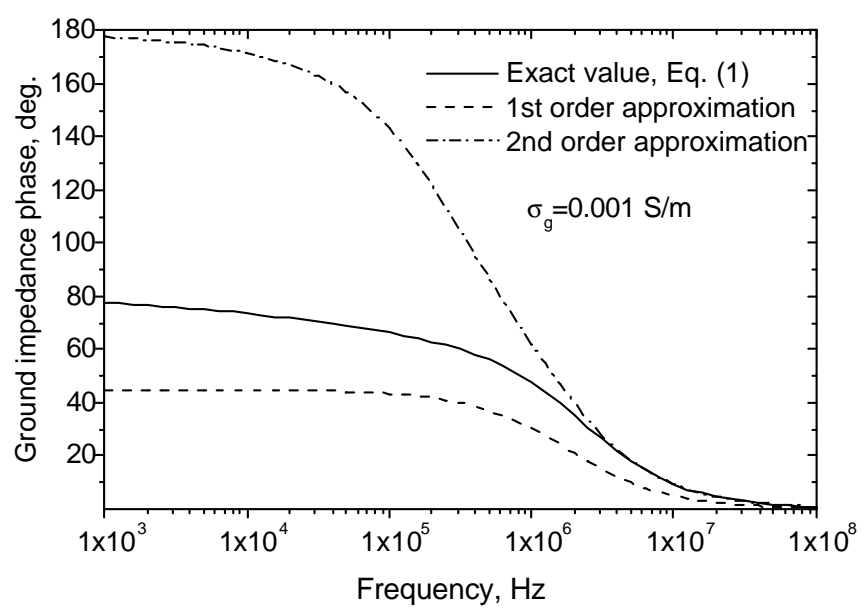

Figure 3a. Ground impedance phase versus frequency by Eq. (1) and (24) $\left(1^{\text {st }}\right.$ and $2^{\text {nd }}$ order approximations); $\varepsilon / \varepsilon_{g}=10, \sigma_{g}=0.001 \mathrm{~S} / \mathbf{m}, h=10 \mathbf{m}$.

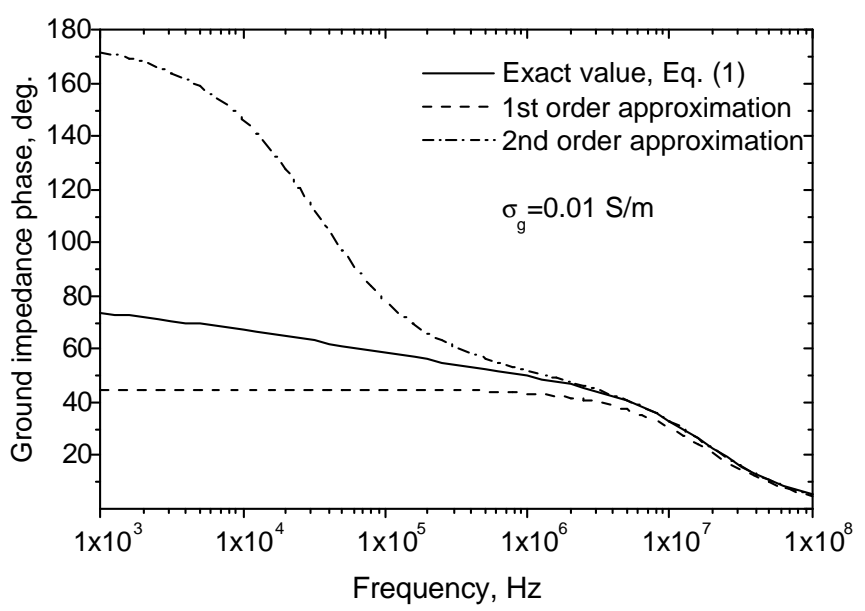

Figure 3b. Ground impedance phase versus frequency, $\sigma_{g}=0.01 \mathrm{~S} / \mathrm{m}$.

accurate approximation in that region. Thus, we can recommend the following compound approximation:

$$
\xi(t) \approx\left\{\begin{array}{cc}
E q .(25), & t \leq t_{0} \\
E q .(8), & t>t_{0}
\end{array}\right.
$$

It should be noted that the numerical computation using FFT requires for a large number of samples (typically, around $10^{6}$ or more) to obtain accurate results. Thus, the approximation above may provide substantial reduction in computational complexity.

\section{Comment On the Transmission Line APProximation}

The early-time behavior of the ground transient given by Eq. (25) is valid within the transmission line approximation, which is itself a low-frequency approximation. It is valid 


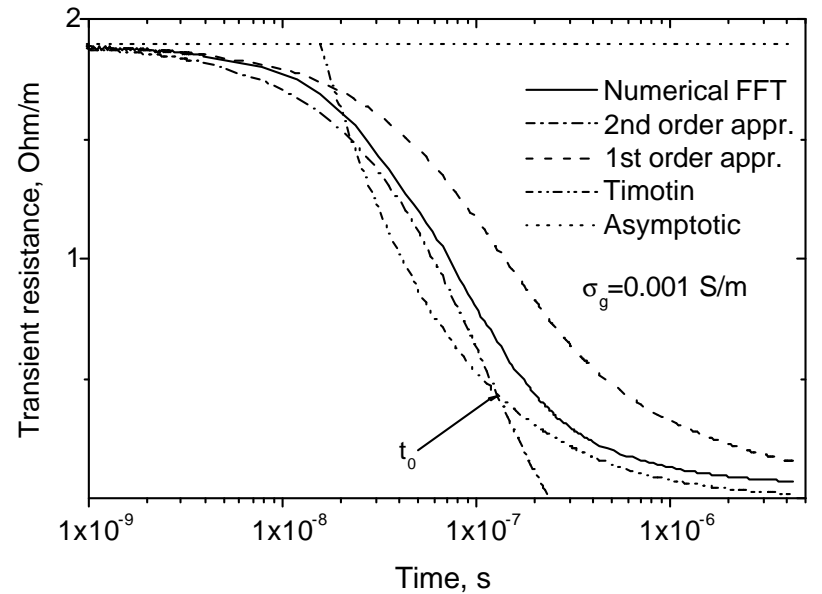

Figure 4a. Ground transient resistance using numerical FFT computation $((1)$ and $(5)), 2^{\text {nd }}$ and $1^{\text {st }}$ order approximations (eq. (25)), Timotin's formula (eq. (8)) and the asymptotic value (eq. (17)); $\varepsilon / \varepsilon_{g}=10, \sigma_{g}=0.001 \mathrm{~S} / \mathbf{m}$, $h=10 \mathbf{m}$.

when the wavelength is higher than the system's transverse dimension (the wire's height),

$$
\lambda>\lambda_{T L}=h,
$$

which can be transformed to

$$
\omega<\omega_{T L}=\frac{1}{h \sqrt{\mu_{0} \varepsilon_{0}}}
$$

where $\varepsilon_{0}$ - is the free space permittivity. In the time domain, the inequality above is

$$
t>t_{T L}=h \sqrt{\mu_{0} \varepsilon_{0}}
$$

Thus, strictly speaking, Eq. (25) is valid for $t_{T L} \leq t<t_{\lim }$. Over the interval $0 \leq t<t_{T L}$ the radiation effects may be of importance and this issue requires for further investigation. The same holds true for (17), which is also valid within the transmission line approximation.

\section{CONCLUSION}

In this paper, we have considered the early-time behaviour of the ground transient resistance of overhead lines. Using the integral representation of the ground impedance in the frequency domain (within the transmission line approximation), we have derived approximate closed-form expression for the transient resistance in the time domain. Keeping in mind that the numerical computations using an FFT algorithm require for a large number of samples (typically, few millions), these approximations may provide substantial reduction in computational power. Additionally, they provide a physical insight into the early-time behaviour

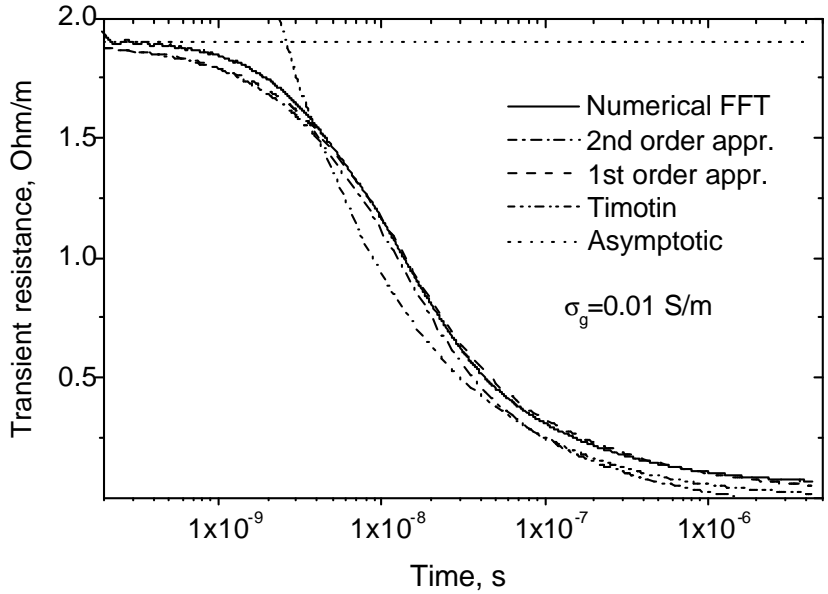

Figure 4b. Ground transient resistance, $\sigma_{g}=0.01 \mathrm{~S} / \mathrm{m}$,

of the transient resistance. When used together with the Timotin's formula, which provides a late-time approximation, they cover the entire range with satisfactory accuracy.

\section{REFERENCES}

[1] F. Tesche, M. Ianoz and T. Karlsson, EMC Analysis Methods and Computational Models, New York: Wiley Interscience, 1997.

[2] F. Rachidi, C.A. Nucci, M. Ianoz and C. Mazzetti, "Influence of a Lossy Ground on Lightning-Induced Voltages on Overhead Lines", IEEE Trans. Electromag. Compat., vol. 38, pp. 250-264, Aug. 1996.

[3] A. Timotin, "Longitudinal transient parameters of a unifilar line with ground return", Rev. Roum. Sc. Tech., Electrotech. Energie, vol.12, no.4, pp.523-535, Bucarest, 1967.

[4] S.L. Loyka, "On calculation of the ground transient resistance of overhead lines", IEEE Trans. on EMC, vol. 41, no. 3, Aug. 1999, pp. 193-195

[5] F. Rachidi, C.A. Nucci, M. Ianoz, Transient Analysis of Multiconductor Lines Above a Lossy Ground, IEEE Trans. Power Delivery, vol. 14, No. 1, Jan. 1999, pp.294-302.

[6] D. Orzan, "Time-Domain Low Frequency Approximation for the Off-Diagonal Terms of the Ground Impedance Matrix", IEEE Trans. Electromag. Compat., vol. 39, pp. 64, Feb. 1997.

[7] G.A. Korn, T.M. Korn, Mathematical Handbook, McGraw-Hill, New York, 1968. 